\title{
A RARE PRESENTATION OF HIV IN NEWBORN - OSTEOMYELITIS
}

\author{
Ashutosh Kumar Sinha1, Abhay Kumar², Sonali Suman³, Smita Kumari ${ }^{4}$
}

${ }^{1}$ Assistant Professor, Department of Cardiology, NMCH.

${ }^{2}$ Associate Professor, Department of Paediatrics, MGM Medical College, Kishanganj.

${ }^{3}$ Senior Resident, Department of Paediatrics, MGM Medical College, Kishanganj.

${ }^{4}$ Senior Resident, Department of Obstetrics and Gynaecology, MGM Medical College, Kishanganj.

ABSTRACT

\section{BACKGROUND}

The Human Immunodeficiency Virus (HIV) is a lentivirus (retrovirus) that causes HIV infection and over time Acquired Immunodeficiency Syndrome (AIDS). AIDS is a condition in humans in which there is progressive failure of immune system, which allows life-threatening opportunistic infections and cancers to thrive. Globally, an estimated 43 million people are infected with HIV. Worldwide, of people living with HIV, the percentage of women with HIV remains at $50 \%$. With maternal diagnostic and prophylaxis during perinatal period, perinatal HIV transmission can be prevented. For this HIV testing and antiretroviral (ARV) prophylaxis and treatment are essential. In our case report, we present a case of preterm baby with HIV seropositivity that was vertically transmitted and accompanied by osteomyelitis. We report this case to emphasise the importance of early detection and early ARV chemotherapy to prevent vertical transmission.

\section{KEYWORDS}

Neonatal Osteomyelitis, ARV Chemotherapy, Vertically Transmitted.

HOW TO CITE THIS ARTICLE: Sinha AK, Kumar A, Suman S, et al. A rare presentation of HIV in newborn - osteomyelitis. J. Evolution Med. Dent. Sci. 2016;5(97):7155-7157, DOI: 10. 14260/jemds/2016/1619

\begin{abstract}
BACKGROUND
Neonatal osteomyelitis in HIV positive neonates is a rare and challenging diagnosis, particularly in early onset period. Osteoarticular infections, although uncommon, represent a severe condition in HIV positive neonates. Neonatal osteomyelitis is predominantly caused by staphylococcus aureus with single bone lesion. Lower extremity joints are commonly affected. Early diagnosis and treatment to reduce the sequelae is very important. Diagnosis involves appropriate radiographic studies and culture of blood and material obtained from infected bone by needle aspirate. Treatment requires prolonged intravenous therapy with antibiotics that achieve high bone penetration and are directed against the identified or presumed causative bacteria and Antiretroviral (ARV) chemotherapy. Here, we report a case of neonatal osteomyelitis in a preterm baby with HIV seropositivity that was vertically transmitted.
\end{abstract}

\section{CASE REPORT}

A 32-week-old, male baby with an Apgar score of 8/9 was delivered normally by a 28 -year-old unbooked primi gravida who presented to obstetric emergency in labour. No antenatal care and investigations were done before. Baby cried immediately after birth, weight was 1.504 gms, vitals were normal, systemic examination unremarkable, no obvious congenital malformation was noted. The neonate was admitted in NICU for preterm care and low birth weight.

Financial or Other, Competing Interest: None.

Submission 25-09-2016, Peer Review 22-11-2016,

Acceptance 29-11-2016, Published 05-12-2016.

Corresponding Author:

Dr. Ashutosh Kumar Sinha,

o/63, Doctors' Colony,

Kankerbagh,

Patna-800020,

Bihar.

E-mail: sonali.suman@gmail.com

DOI: 10. 14260/jemds/2016/1619
At 29 hours of life, the baby developed respiratory distress. Sepsis screen was positive (Micro ESR $=8 \mathrm{~mm}$ at 1 hour, CRP $=50 \mathrm{mg} / \mathrm{L}) \mathrm{Hb}=16.4 \mathrm{~g} / \mathrm{dL}, \mathrm{TLC}=16000 / \mathrm{mm} 3$, platelets $=$ $200000 / \mathrm{mm} 3$. Chest $\mathrm{x}$-ray was done, which showed bilateral infiltrates. Early onset neonatal sepsis was diagnosed and IV amoxicillin/clavulanic, amikacin, 02 therapy and IV fluids were given. HIV screening of parents were done. Both mother and father were tested positive for HIV-1. Mother CD4 count was $180 / \mathrm{mm} 3$. Screening for hepatitis, syphilis and TORCH was negative. Both were referred to ART Centre for HAART.

Baby developed DIC at $94 \mathrm{hrs}$. of life which manifested as gastrointestinal bleed, (PT/PTT - prolonged, D-dimer = positive, platelet $=40,000 / \mathrm{mm} 3$ ), for which treatment was given (vitamin $\mathrm{K}$ injection, FFP, platelet transfusion, total parenteral nutrition - amino acid, intralipid and MVI). Later on at $120 \mathrm{hrs}$. of life baby developed acute non-oliguric renal failure (blood urea $=87 \mathrm{mg} / \mathrm{dL}$, serum creatinine $=1.7$ $\mathrm{mg} / \mathrm{dL}$ ), which was managed conservatively. Amino acid concentration was reduced and antibiotics was changed to piperacillin-tazobactam and cefotaxime. Investigations were repeated on day 6 . Total leucocyte count $=11000 / \mathrm{mm} 3$ with CD 4 count of $25 \%$. Sepsis screen was still positive. Blood culture sent on day 1 and day 6 was sterile. Apparent improvement was noted after 2 weeks. Activity was better, tolerated feeds well, renal profile normalised. Antibiotics were stopped.

On day 20, swelling of both thighs (left > right) with decreased and painful limb movement of both legs were noted. $\mathrm{X}$-ray revealed gross osteomyelitis with massive periosteal reaction involving both femurs and left tibia. Ultrasonography of limbs revealed marked subcutaneous cellulitis with thickening and increase echogenicity of subcutaneous fat. Irregular cortical bone surface was present along with periosteal reaction. Doppler examination of both lower limbs was normal. Ceftriaxone sodium was started. Blood culture sent on day 22 revealed growth of staphylococcus aureus sensitive to vancomycin, clindamycin and trimethoprim- 
sulfamethoxazole. IV vancomycin was started and continued for 3 weeks.

Baby responded well to the treatment and condition of baby improved during hospital stay, which was evident by decrease in the swelling and improvement of movements. Baby was discharged on $50^{\text {th }}$ day. DNA PCR done on day 40 and repeated on day 43 were positive for HIV-1, CD4 count was $25 \%$, classified as HIV clinical disease stage 3 with advanced immunosuppression [WHO 2007]. Antiretroviral therapy (HAART; zidovudine + lamivudine + nevirapine) was started on day 43 along with Pneumocystic jirovecii pneumonia prophylaxis with cotrimoxazole.

On followup - last followup was at 15 months, growth and development of baby was satisfactory. There was no limb length discrepancy or angular deformity and the child was able to bear full weight without support.

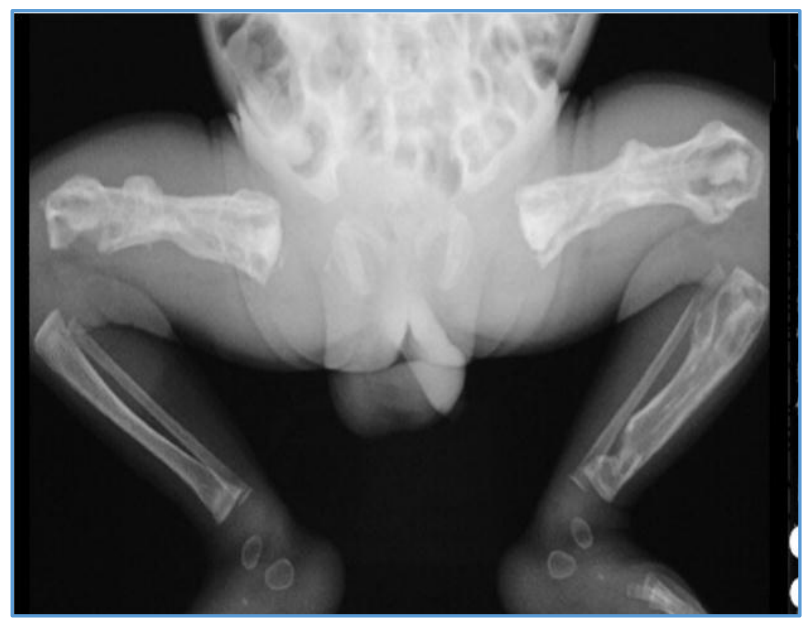

Figure 1.X-Ray showing Gross Osteomyelitis + Periosteal Reaction (Involves Both Femur and Left Tibia)

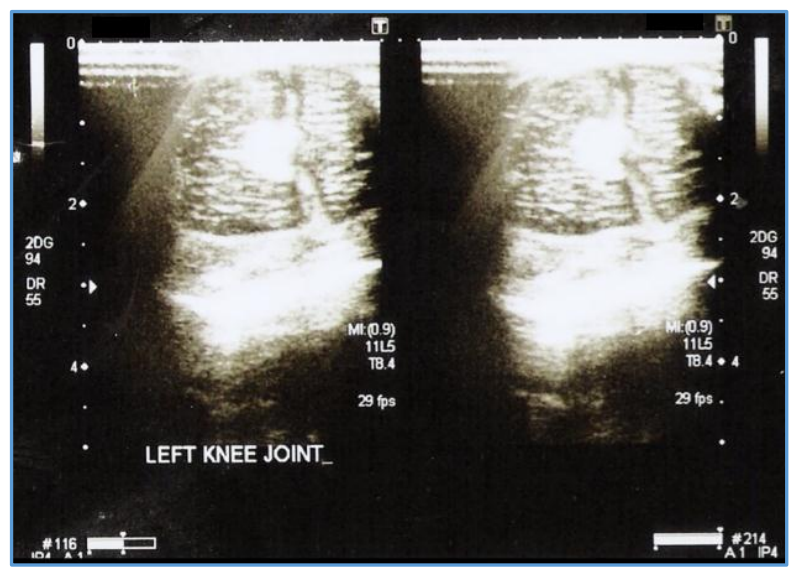

Figure 2. Ultrasonography of Both Lower Limbs - shows Marked Subcutaneous Cellulitis, Irregular Cortical Bone Surface and Periosteal Reaction

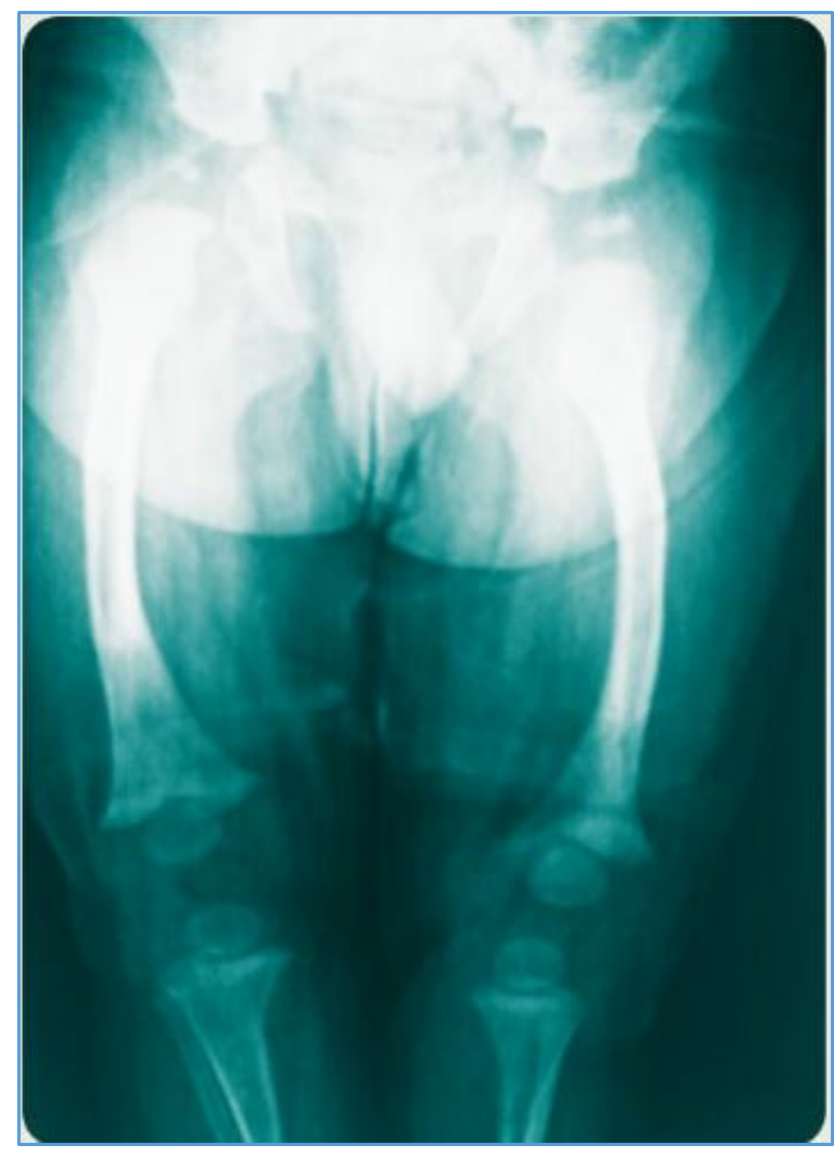

Figure 3. X-Ray at 4.5 Months showing Radiological Healing of Both Femurs

\section{DISCUSSION}

HIV infection is a serious condition with high mortality. HIV cases are increasing due to difficulty in diagnosis, long latent period and routes of transmission. We have reported this case to show the importance of universal HIV screening for all pregnant women, importance of antiretroviral therapy, safe delivery practices and modified infant feeding.

The overall incidence rate for bone and joint infections is 0.12 per 1000 live births and 0.67 per 1000 neonatal intensive care (NICU) admissions(1) with a mortality rate of $7.3 \%$.(2) The most common bacterial pathogen causing osteomyelitis in children is staphylococcus aureus in all age groups.(3) Group B streptococcus (streptococcus agalactiae) and gram negative organism (E. coli and Klebsiella pneumoniae) are also important in neonatal period.(4) The most commonly affected joints are the Hip joints (31\%), Knee joints (25\%) and Ankle joints $(18 \%) .{ }^{(5)}$ If the mother is infected with HIV, the foetus has a $25 \%$ chance of being infected by the virus. Upto $50 \%$ of infants get HIV from their mother late in pregnancy or during delivery.

Riordan et al has reported osteomyelitis caused by streptococcus pneumoniae 9S, resistant to penicillin in a 6month-old girl with HIV by PTCT (Parent To Child Transmission).(6) Yao et al have reported osteomyelitis in $0.9 \%$ of a retrospective record review of 888 adult patients with HIV.(7) 
Mother To Child Transmission (MTCT) is the most important source of Human Immunodeficiency Virus (HIV) in children below the age of 15 years. The rate of perinatal transmission is $15 \%-25 \%$ in developed countries and $25 \%$ $45 \%$ in developing countries.(8) The rate of perinatal transmission without intervention is 19\% - 36\%(9); $25 \%$ - 30\% of total transmission occurs during the prenatal period, mainly in late pregnancy(10) while $70 \%-75 \%$ of total transmission occurs during the intranatal period. Postnatal transmission is via breast milk and accounts of $10 \%-16 \%$ of all transmission.(11) In 1994 Paediatric AIDS Clinical Trial Group (PACTG) Protocol, 076 demonstrated that the administration of zidovudine during pregnancy and labour and then to newborn decreased the risk of perinatal transmission of HIV by $68 \%$ from $25.5 \%$ to $8.3 \%$.(12) Children born to seropositive mother should be followed closely during the first several months of life for evidence of HIV infection, so that optimal care can be provided.

Prevention of HIV infection is one of the priorities of all health strategies, especially those related to maternal and child health. Educational programmes should seek to explain the risk behaviour associated with HIV infection, the implications of HIV infection in pregnancy outcome and the ways in which the risk of HIV infection can be reduced. HIV infection should be identified prior to pregnancy or as early as possible. This provides the best opportunity to prevent infant HIV infection and to identify and start therapy as soon as possible in infants who become infected.

\section{CONCLUSION}

Neonatal osteomyelitis in HIV positive infant, although a rare presentation remains a diagnostic and therapeutic challenge. Osteomyelitis should be considered in newborn infants presenting with clinical signs of sepsis without any obvious focus. This is essential for early diagnosis and treatment to prevent any long-term morbidity.

\section{REFERENCES}

1. Ho NK, Low YP, See HF. Septic arthritis in the newborn-a 17 years clinical experience. Singapore Med J 1989; 30(4):356-8.
2. Caksen H, Ozturk MK, Uzum K, et al. Septic arthritis in childhood. Pediatr Int 2000;42(5):534-40.

3. McPherson DM. Osteomyelitis in the neonate. Neonatal Netw 2002;21(1):9-22.

4. Liao SL, Lai SH, Lin TY, et al. Premature rupture of membrane: a cause for neonatal octeomyelitis? Am J Perinatol 2005;22(2):63-6.

5. Halder D, Seng QB, Malik AS, et al. Neonatal septic arthritis. Southeast Asian J Trop Med Public Health 1996;27(3):600-5.

6. Riordan Al, Adalat S, Graham C. Successful treatment with azithromycin and rifampicin in penicillin and cephalosporin insensitive pneumococcal osteomyelitis in a child with HIV infection: a case report. Cases J 2008;1(1):283.

7. Yao Q, Frank M, Glynn M, et al. Rheumatic manifestation in HIV-1 infected patients and literature review. Clin Exp Rheumatol 2008;26(5):799-806.

8. Guidelines for the Prevention of Mother to Child transmission of HIV. National AIDS Control Organisation. Available from: http:www.nacoonline.org/guideline/guideline_9pdf.

9. Kapoor A, Kapoor A, Vani SN. Prevention of mother to child transmission of HIV. Indian J Pediatr 2004;71: 247-51.

10. Ehrnst A, Lindgren S, Dictor $M$, et al. HIV in pregnant women and their offsprings: evidence for late transmission. Lancet 1991;338(8761):203-7.

11. Nduati R, John G, Mbori-Ngacha D, et al. Effect of breastfeeding and formula feeding on transmission of HIV-1: a randomized clinical trial. JAMA 2000; 283(9): 1167-74.

12. Connor EM, Sperling RS, Gelber RR, et al. Reduction of maternal-infant transmission of human immunodeficiency virus type 1 with zidovudine treatment. Pediatric AIDS clinical trials group protocol 076 study group. The New England Journal of Medicine 1994;331(18):1173-80. 